Article

\title{
Consequences of Cervical Cancer Treatment on Sexual Health in Chinese Cancer Survivors: A Qualitative Study
}

\author{
Sha Ye ${ }^{1}$, Xiangcheng Yang 2 , Guoping $\mathrm{He}^{3}$, Peter A. Maciek ${ }^{3}$, Wenjuan Zhou ${ }^{3, *}$ \\ 1 Hunan Cancer Hospital and the Affiliated Cancer Hospital of Xiangya School of Medicine Central, the South University, \\ Changsha, Hunan, China \\ 2 Zhuzhou Central Hospital and the Affiliated Zhuzhou Hospital of Xiangya School of Medicine Central South University, \\ Zhuzhou, Hunan, China \\ 3 Xiangya School of Nurse, the Central South University, Changsha, Hunan, China
}

Received July 4, 2018; Accepted October 16, 2018

\begin{abstract}
Background: The attitudes and perceptions of cervical cancer survivors (CCS) toward sexual activity subsequent to a diagnosis of cervical cancer and its treatment are unknown. This study describes the experience of CCS in Hunan Chinese in relation to sexuality and sexual function after cervical cancer treatment.

Methods: We used descriptive phenomenology to qualitatively assess these experiences. Purposive sampling was used to recruit 20 CCS. Data were collected through in-depth interviews and analyzed according to Colaizzi's method to explore the essence of the experience in sexuality among CCS after cancer treatment.

Results: Uncertainty, fear, and worry dominated the attitudes and behaviors of CCS-related to sexual activity after treatment. Four themes explain these complex emotional responses: 1) needing information; 2) dealing with sexual changes physiccally and emotionally; 3) communicating with a partner; 4) attribution of fault to her one-lifetime sexual partner.

Conclusion: Sexual life was influenced by the physical changes associated with cervical cancer treatment. These changes in combination with inadequate information and limited communication led to uncertainty, fear and worry about engaging in sexual activity and relationship distress. Chinese CCS need targeted and patient-centered information on the change in sexual life caused by treatment, anticipatory guidance, and support in communication with providers and partners, and strategies to cope with the physical and psycho-sexual sequelae of treatment, all of which must be congruent with their cultural norms.
\end{abstract}

\section{KEYWORDS}

Cervical cancer survivors; sexuality; sexual health; phenomenology; quality of life

\section{INTRODUCTION}

Cervical cancer is the second most common cancer in women in less developed countries and is the fifth most common cancer in women worldwide. In 2012 there were nearly 530,000 new cases of cervical cancer patients in the world [1]. There are 60,000 new cases diagnosed with cervical cancer each year in China, which account for about 29\% of the world's total new incidence [2]. The screening and early detection initiative through the "National Cervical Cancer Screening Program" survivorship [3]. However, the majority of women in China diagnosed with cervical cancer receive surgery, often combined with radiotherapy and/or chemotherapy. The side effects of multimodality therapy can adversely affect sexuality and sexual function, resulting in emotional and marital relationship distress $[4,5]$.

\footnotetext{
${ }^{*}$ Correspondence: Wenjuan Zhou, E-mail: wenddi.zhou@csu.edu.cn.
}

Sexuality is an inherent component of a woman's quality of life and sexual health issues after gynecologic cancer treatment which can profoundly affect the quality of life in the ensuing survivorship [6-8]. Cervical cancer survivors (CCS) had a significant decline in sexual functions compared with healthy people [9]. Surgery can alter sexual organs; surgery and chemotherapy can induce premature menopause, and radiotherapy can damage the vaginal mucosa often resulting in stenosis and fibrosis [4]. Cancer treatment-induced menopause produces adverse effects including but not limited to vasomotor symptoms, vaginal atrophy, painful intercourse, decreased libido and arousal and changes in body image. Nearly half of cervical cancer survivors are at risk for sexual dysfunction due to the menopausal symptom profile $[8,10]$. The psychological sequelae may include anxiety, fear of performance, guilt, depression, concerns about appearance and worry about cancer recurence. 
Studies on the sexuality of cervical cancer patients in China have predominantly focused on the effects of surgical treatment $[11,12]$. The attitudes toward sexuality among the CCS in China and how they adapt to post-treatment changes in sexual function are relatively unknown. Culture is an important consideration in understanding a woman's experience related to sexuality [5]. In general, Chinese women have more conservative attitudes, beliefs, and behaviors toward sexuality compared to women in Western society $[13,14]$. Chinese women often are uncomfortable with intimate issues, and there is an accepted silence about discussing sexuality in traditional Chinese families [15]. We have found no qualitative studies that describe the experience of sexual function after cervical cancer treatment fro$\mathrm{m}$ the perspective of Chinese women. The purpose of this present study was to describe and understand the experience of sexual health after cervical cancer treatment in women from Hunan, China.

\section{METHODS}

\section{Research design}

Descriptive phenomenology is a qualitative method to understand the essence of the life experience for persons who have experienced the phenomenon of interest. Colaizzi's methodological framework was used to guide this study [16]. This framework offers a holistic view of objective and subjective realities and provides direction and guidance to capture the complexity of people's life experiences. The goal is to gain an understanding of the experience through written and verbal description, identifying the distinct and critical components of the phenomenon [17].

\section{Study participants}

The participants were recruited from the regional cancer center of Hunan province and with the largest gynecologic oncology department in Hunan, China. The physicians in the Outpatient Department of the Hunan Cancer Hospital assisted in the study by identifying and recommending qualified survivors who returned to the hospital for a follow-up examination. Eligible participants diagnosed with cervical cancer met the following criteria: >18 years old; stage I, II, or III cervical carcinoma with within 3 months to 3 years post-treatment; no severe mental disorder or dementia; no current evidence of cancer recurrence; able to read, write, and speak Chinese; willing to provide informed consent. Purposive sampling was used to identify those women who experience the phenomenon of interest and who had the potential to be rich informants. Demographic characteristics of all participants were shown in Table 1.

\section{Procedure}

Semi-structured interviews were used to collect qualitative data from participants. The interview guide consisted of several open-ended questions (Table 2). Researchers recorded the participants' non-verbal reactions during interviews and took notes of critical Information supplied by participants. After each interview, detailed notes of the interview process, including thoughts, feelings, and responses were recorded. All the interviews were conducted by the same investigator [WZ] in a private office of the gynecologic oncology clinic in the regional cancer center of Hunan province where confidentiality and privacy could be maintained. The interviews lasted 30 to $60 \mathrm{~min}$ and were audiotaped. Interviews were then transcribed verbatim.

Table 1. Demographic Characteristics of Participants $(n=20)$.

\begin{tabular}{lrr}
\hline Characteristic & $n$ & $\%$ \\
\hline Age & 7 & 35 \\
$30-40$ & 10 & 50 \\
$41-50$ & 3 & 15 \\
$51-60$ & & \\
Marital Status & 17 & 85 \\
Married & 3 & 15 \\
Divorced & & \\
Education & 2 & 10 \\
College & 15 & 75 \\
High school & 2 & 10 \\
Primary school & 1 & 5 \\
Illiterate & & \\
Residence & 18 & 90 \\
Rural resident & 2 & 10 \\
Urban resident & & \\
Occupation & 6 & 30 \\
Housewife & 4 & 5 \\
Freelance & 1 & 5 \\
Village cadre & 8 & \\
Farmer & 1 & \\
Worker-employed & & \\
\hline
\end{tabular}

\section{Ethical considerations}

The Institutional Review Board of the regional cancer center and the academic institution approved our study. The informed consent was signed after participants were informed and stated that they fully understood. All participants were voluntary and anonymous, and they could stop the interview at any time without any influence on their care. Participant names were removed and replaced by coded numbers. All data were locked and accessed only by the investigator who had conducted the interviews (WZ).

\section{Data analysis}

After each interview, the transcriptions of recordings were reviewed noting any pause, changes of tone, sighing, sobling, laughing and those were marked on the transcribed interviews. Field notes and observations were also integrated throughout the analysis. Data were entered into the qualitative software program Nvivo 10.0 for data management. The analysis was witnessed by instructors as well as transcribed interviews and data analysis was performed according to the seven-step procedure established by Colaizzi (1978) [16]. Those steps included: 1) reading and re-reading in order to obtain a general sense about the whole content, 2) after reviewing the transcripts, significant statements/phrases relating to the experiences under research were extracted, 3 ) formulation of meanings from these significant statements, 4) sorting, meanings into categories, clusters of themes, and themes, 5) integrating findings into an exhaustive description of the phenomenon under study, 6) describing the fundamental structure of the phenomenon and 7) validating the findings from the research participants to compare the researcher's descriptive 
results with their experiences. The transcripts of interviews were returned to participants for verification of authenticity, accuracy, and completeness of information. In subjects' participants acknowledged and verified that the transcript was correct, that the analyses were correct and the participants had accepted that these documents were accurate.

Table 2. Interview Guide.

Please tell me did cervical cancer treatments affect your sexual life? If yes, how?

- Probe 1: How long after the end of cancer treatments, did you resume your sexual life? How did you get the information, which helped you make the decision to resume your sexual life?

- Probe 2: Before cancer treatments, did you consider their impacts on your sexual life? If yes, how?

Did the disease affect your self-perception? If yes, how?

Did the disease affect your body image? If yes, how?

Did cancer treatments affect the communication between you and your partner? If yes, how?

- Probe 3: How did your partner communicate with you about those changes of your sexual life?

Did cancer treatment affect your ability to be intimate sexually? If yes, how?

\section{RESULTS}

\section{Themes}

The findings of this study suggest that uncertainty, fear, and worry are dominant emotional responses of the CCSrelated to inadequate information about sexual health after treatment, physical changes and symptoms and communication with partners, all of which negatively affected the CCS' sexuality, sexual function and willingness to engage in sexual activity and intimacy. Four themes described the essence of the phenomenon of sexuality and sexual function after cervical cancer treatment: needing information, dealing with sexual changes-physically and psychologically, communicating with the partner, and attribution of fault to her one-lifetime sexual partner.

\section{Needing information}

A few participants gained information from formal sources, such as health care providers or educational materials.

"I have read some books; I know that appropriate sexual life can improve physical and mental health. At the return visit, a patient inquired the provider, and the provider said that appropriate sex was beneficial to her body and mind." (Age 37, divorced, rural resident, high school education, freelance)

However, informal information channels profoundly influenced many participants as personal experiences and myths perpetuated by friends or family members within their culture. These generated fear, anxiety, and worry and negatively contributed to sexual function decision behaviors.

"My aunt's sister who got uterus surgery at 17 years old, and then she got married. I thought if she got married, she must have a sexual life." (Age 46, married, rural resident, high school education, freelance)

"I heard folk people said that I should not have too much sex, and should not be together with a sexual partner too frequently. People told me that for a long lifetime, my partner should not stay with me, and we should be apart from each other." (Age 41, married, rural resident, high school education, farmer)

"I have a little fear of sex. When I found this disease, my husband and I slept together and the bleeding happened. For this kind of reasons, I am very scared of sex." (Age 45, married, rural resident, high school education, housewife)

\section{Dealing with sexual changes-physical and psycho-} logical responses

Participants described many adverse alterations in their sexual health that influenced sexual function, activity, and sexual responsiveness. Sexual desire and arousal were diminished, menopause symptoms were common including vaginal dryness, painful intercourse, and loss of pleasure, satisfaction and orgasmic ability. Further, the sexual and reproductive side effects resulting from treatment for cervical cancer include premature menopause, loss of fertility, and a reduction in vaginal elasticity, often resulting in pain and impaired sexual function.

"After surgery, my abdomen was all cut off, so it is empty inside now. When my husband had sex with me, he said that it's empty. Sometimes, when I walk, hike or step for a long time, I feel really painful, something inside falling down, and uncomfortable inside." (Age 41, married, rural resident, high school education, housewife)

Body image played an important role in CCS sexual health. For example, the surgical scar had a substantial negative impact, created emotional distress and resulted in worry about a negative partner response during sexual activity.

"There is a huge scar in my belly, at least 15 to $25 \mathrm{~cm}$. Frequently, it is itching, and I will scratch. Many times, scratching makes me upset and annoyed, and I will lose my temper to my husband. I would show my belly to my husband, but I was afraid that my husband would see me ugly. Sometimes I would stop him to see the scar. I was afraid that he would avoid me, dislike me." (Age 41, married, rural resident, high school education, housewife)

Fear of cancer recurrence was a universal concern expressed by participants. The majority of Chinese cervical cancer survivors lacked accurate information and believed that engaging in sexual activity could be related to the relapse of cancer or genital infection. 
"I heard some women who got this disease said, if my husband and I sleep together, I should be cautious of inflammation. I was scared when heard these. Sometimes I really want sex, but feel scared. If by any chance acident happens, I will die." (Age 44, divorced, rural resident, primary education, farmer)

The physical changes because of cervical cancer treatment resulted in physical and psychological distress and a high degree of sexual dysfunction and relationship strain.

\section{Communicating with partners}

Women appeared to have limited communication with partners, little insight into how the partner was coping was offered, and the CCS seemed to make decisions on her own about engaging in sexual activity. We found many couples can cope with the cancer diagnosis and treatment, and were able to draw on support from each other and their families. There was also evidence of the partner's concern for the woman's health as a priority over sexual function.

"He said he would respect my feelings. I asked him what if my conditions would not endure sex. He said he would follow my willing. He said he would respect my choice, and his only wish was that I got a better lifebetter to my health." (Age 34, married, rural resident, high school education, housewife)

"He is afraid of that if someday I die, he would have no such companion anymore. He is thinking for the sake of daily life, for a company, for reliance. Even there is no talking between us, getting the sight of me is a routine of his life." (Age 45, married, urban resident, high school education, freelance)

To some couples, changes caused by cancer treatment can lead to the end of their relationship. Infertility is a consequence of hysterectomy, which the majority of participants had as well as chemotherapy. For two participants, infertility caused significant distress and regretted for the treatment decision due to divorce.

"While he wanted to divorce, I indeed did not. I think that I would have a better life without this disease. I always feel that if I have no cervical cancer surgery and I still have the ability to give birth, I may want a marriage. But I have no ability now and have this disease with me, I won't consider getting married." (Age 37, divorced, rural resident, college education, freelance, first divorce due to infidelity of an ex-husband and second divorce due to loss of fertility caused by cancer treatment)

We also found that some survivors feared to be unable to meet the partner's sexual needs. Concerns about performance and providing an adequate sexual life and satisfaction for the partner transformed how the participant viewed loyalty in their relationship. Moreover, some even considered the idea of letting the husband go out and find another woman as a buffer to their inability to resume sexual life.

"Sometimes, he bothered me for sex. I was annoyed and repulsed him. I was annoyed but afraid of speaking out. Because I was upset, I got an idea in mind like, 'You go out! You go out to find other women! I will not care.' However, I didn't dare to speak out. I was afraid that he really went out to find other women. Honestly, I didn't want to let him go." (Age 41, married, rural resident, high school education, farmer)

\section{Attribution of fault to her one-lifetime sexual partner}

In addition to the relationship strain, some women accused her partner of giving her disease. Generally, those women only had one sexual partner in her whole life. After diagnosis and treatment, they were aware of cervical cancer risk factors, so they accused their only partner as the cause of the disease.

"I am well-behaved. I have no affairs in my life. However, my husband was a truck driver. I had heard of gossips about his affairs. I had fights with him on this issue. Even I showed some evidence I found; he did not admit. I believe the only possible cause of my disease is my husband's unclear behaviors." (Age 46, married, rural resident, high school education, freelance)

Those women developed negative feelings towards their partner which could influence their sexual life after cancer treatments. Those feelings can also be worsened by the side effects of cancer treatments and fears of cancer recurrence.

"Because of the fear and anger from my disease and his behaviors, I feel hard to be intimate with him. Every time we had sex, I recalled his affairs, and I lost feelings. I blamed him that he is the one killing me." (Age 46, married, rural resident, high school education, freelance)

Even some of them considered to divorce, but most of them would not make the decision of divorce, because culture and social norms bound traditional marriage. For those women, divorce not only ends relationships in a family but also affects their social functions in their community.

"In my opinion, if you are married, you have to remain to that family for the rest of your life. It is a great shame for a woman to divorce her husband. So I chose to stay with my husband, although we had bad feelings in a sexual relationship." (Age 47, married, rural resident, high school education, farmer)

\section{DISCUSSIONS}

Cancer-related sexual problems have a complicated relationship with biologic, inter-personal, psychological, and social- cultural factors [5]. The findings of this study confirm that those factors are integrally related and result in physical and psychological distress and decreased the quality of life $[4,7,18]$. Sexual health is recognized as vital to the quality of life for gynecologic cancer survivors $[7,8]$. The themes in this study of inadequate information, dealing with sexual changes, communicating with partner, and attribution of fault to her one partner were associated with emotionally distressful responses related to the decreased quality of life. In contrast to many studies published with cancer survivors in the western world, the influence of Chinese 
culture was significant and must be integrated into all interventions designed for Chinese CCS [5].

In contrast to a study with British CCS who reported wanting more discussion and sex information from their providers [19], the Chinese CCS in our study did not initiate such conversations with their providers. In CCS communication and relationship with their physicians, it is very rare to discuss sex topics as an influence of Chinese cultures and norms. Inquiries about sex are communicated obliquely as "Is sleeping together allowed?" and "Will this behavior cause problems?" Myths, reliance on personal experiences, and the Chinese cultural influence related to sexual attitudes were dominant factors in the experience for participants in our study. Traditional held Chinese beliefs about sex suggest that sexual life can damage one's energy and frequent sexual activity may be harmful to health $[13,14]$. Fear related to sexual activity was also a common emotional response of participants in our study.

Education supplied by health providers about physical changes and strategies to manage the consequences of those changes is a critical intervention to manage symptoms and to dispel myths associated with the consequence $[8,18]$. However, the best way of addressing and discussing sexual issues has not yet been well established in China and most likely are predominated by the traditional environment surrounding discussion of sexual function with patients, especially in the rural regions of China where traditional cultures and norms are highly influential. Changing personal beliefs and community culture could help develop strategies to deal with sexuality issues and create a basis for the rehabilitation professional to provide a supportive and safe environment to assist the CCS for their needs regarding sex after treatment. Once communities understand the meaning and importacne of sexual health to overall health, like sex after cervical cancer treatments, providers will be better able to talk to patients about sex openly.

Partners of gynecologic cancer survivors are deeply affected by the change in their sexual lives and often have strong and conflicting emotions of worry about the woman's health, desire for sexual activity and some, guilt for their desires of sex and intimacy [7]. There was evidence of these responses in our study but also concerns about not meeting the sexual needs of the partner and the combined inadequate knowledge, lack of strategies to manage symptoms and limited partner communication often resulted in the CCS deciding to restrain, avoid or reject sexual activity. In a study conducted in Australia with cervical cancer survivors, couples were able to talk about sexual life openly and frankly [19], in contrast, our study showed limited or lack of partner communication. Consideration of the sexual partner's loyalty was identified in our participants. In a study with gynecological cancer survivors in Hong Kong, 33\% of participants reported that their husbands had an affair after a cervical cancer diagnosis, while the remaining participants also expressed their worries about that their husbands may have affairs currently or in the future [20]. Psycho-edu- cation interventions including the survivor and the partner can be very successful in stabilizing and maintaining quality partnered relationships and improving the sexuality and intimacy for the couple [4, 7, 8, 18, and 21].

Our study found an instance that some participants accuse their partner's risk behaviors as the cause of their disease. The role of male behavior in cervical carcinogenesis has been reported in many research studies. For women with one lifetime sexual partner, it had reported when husbands had sexual relationships both before and during the marriage, wives' risk of getting cervical cancer increased by 6.9 [22]. Most of our participants are from rural regions which are usually constrained by traditionnal social norms such as arranged marriage, living with husband's family, taking household work, the stigma of divorce, and restrictive moral supervision. Many of them only have a one-lifetime sexual partner-their husband. Meanwhile, those husbands, who have more power and less constraint in the family, usually are the major family economic support. In these traditional Chinese families, disparities of social norms and inequality of labor and power division exist between the two genders, which can increase the risk of transmission of HPV infection from men to women [23].

We found when women had been diagnosed with cervical cancer and learned about its risk factors, some of them would try to trace what was the cause of her case of the disease. For women who have a one-lifetime sexual partner and negative experience of partner's infidelity, they may attribute the fault to their one-lifetime sexual partner, which can worsen their marital relationships and negatively impact their sexual life. Strategies to improve this situation include providing CCS with information about protective sexual behaviors and education in healthy sexual psychology. More importantly, when investigators design sexual health studies in cervical cancer, the social risks for women who participate as study subjects need to be carefully considered.

The purposive sample of 20 participants provided sufficient qualitative data to supply a rich description of their experience. However, it may not have provided an exhaustive description as there were a limited number of women $>50$ years, only two who reported the impact of infertility, the number of years married was not known, and the majority were of relatively low social status. Also, only cervical cancer survivors were interviewed. Interpretation of our data may have been enhanced by data collected through interviews with gynecologic physicians and survivors' partner.

\section{CONCLUSION}

The sexual life of cervical cancer survivors was adversely influenced by the physical changes induced by the cancer treatment and further compromised by the emotional responses, inadequate information, limited or lack of communication, and attribution of fault to her one-lifetime sexual partner. The majority of women in this study were $<50$ years of age and faced with dealing with cancer, premature menopause, and significant surgical changes. 
These women struggle with multiple losses and at the same time are trying to resume a sexual life. In an environment of traditional Chinese culture and the silence of discussing sexuality, it is a medical imperative to develop educational and supportive interventions for Chinese cervical cancer survivors to cope with their sexual life and partner relationships.

\section{CONFLICT OF INTERESTS}

The authors declare that there is no conflict of interest regarding the publication of this paper.

\section{ACKNOWLEDGMENTS}

This study was supported by the Science Foundation for the Youth Scholars of Central South University (No.1249. 17), Hunan Province Philosophy and Social Science Fund (No. 14YBA396), China Medical Board Nursing Faculty Grant (No. 13-168-201607), and Ministry of Education Humanities and Social Sciences Fund (No.17 YJCZH273).

\section{REFERENCES}

1. Torre LA, Bray F, Siegel RL, Ferlay J, Lortet-Tieulent J, Jemal A. Global cancer statistics, 2012. CA Cancer J Clin. 2015; 65(2):87-108.

2. Chen W, Zheng R, Baade PD, Zhang S, Zeng H, Bray F, et al. Cancer statistics in China, 2015. CA Cancer J Clin. 2016; 66(2):115-132.

3. Xinhua News Agency. ACWF vigorously promote the health of women and children have made remarkable achievements [Internet]. 2012 Mar 1. Available from: http:// www.gov.cn/jrzg/2012-03/01/content_2079800.html.

4. Carter J, Stabile C, Gunn A, Sonoda Y. The physical consequences of gynecologic cancer surgery and their impact on sexual, emotional and quality of life issues. J Sex Med. 2013; 10 Suppl 1:21-34.

5. Bober SL, Varela VS. Sexuality in adult cancer survivors: challenges and intervention. J Clin Oncol. 2012; 30(30): 3712-3719.

6. Tierney DK. Sexuality: a quality-of-life issue for cancer survivors. Semin Oncol Nurs. 2008; 24(2): 71-79.

7. Ratner ES, Foran KA, Schwartz PE, Minkin MJ. Sexuality and intimacy after gynecological cancer. Maturitas. 2010; 66: 23-26.

8. Krychman M, Millhesier LS. Sexual health issues in women with cancer. J Sex Med. 2013; 10(Suppl 1):5-15.

9. Lammerink EA, de Bock GH. Pras E, Reyners AK, Mourits MJ. Sexual functioning of cervical cancer survivors: A rev- iew with a female perspective. Maturitas. 2012; 72(4):296304.

10. Hughes MK. Alterations of sexual function in women with cancer. Semin Oncol Nurs 2008; 24:91-101.

11. Zhang X, Gao Y, Yao Y, Yu H, Lu H. Effect of Different Operation Modes on Sex Life in Women Received Hysterectomy. Chin Ment Health J. 2002; 16(11): 749-757.

12. Chen $\mathrm{L}$, Zhang $\mathrm{S}$, Zhang $\mathrm{P}$. To investigate the clinical value of laparoscopic nerve-sparing radical hysterectomy for early cervical cancer. Chin J Pract Gynecol Obstet. 2013; 29(8): 645-648.

13. Higgins L, Zheng M, Liu Y, Sun C. Attitudes to marriage and sexual Behaviors: a survey of gender and culture differences in China and United Kingdom. Sex Roles. 2002; 46(3-4):75-89.

14. So HW, Cheung FM. Review of Chinese sex attitudes and applicability of sex therapy for Chinese couples with sexual dysfunction. J Sex Res. 2005; 42(2):93-101.

15. Chang J. The Tao of love and sex: the ancient Chinese way to ecstasy. New York: Penguin; 1997. p. 34-45.

16. Colaizzi PF. Psychological research as the phenomenologist views it. Valle IS, King M, editors. Existential phenomenological alternatives for psycho-logy. New York: Plenum; 1978. p. 48-71.

17. Linhares $\mathrm{CH}$, The lived experiences of midwives with spirituality in childbirth: mana from heaven. J Midwifery Womens Health. 2012; 57(2):165-171.

18. Barbera L, Fitch M, Adams L, Doyle C, DasGupa T, Blake J. Improving care for women after gynecological cancer: the development of a sexuality clinic. Menopause. 2011;18 (12):1327-1333.

19. Juraskova I, Butow P, Robertson R, Sharpe L, McLeod C, Hacker N. Post-treatment sexual adjustment follo-wing cervical and endometrial cancer: a qualitative insight. Psychooncology. 2003; 12(3): 267-279.

20. Molassiotis A, Chan CW, Yam BM, Chan ES, Lam CS. Life after cancer: adaptation issues faced by Chinese gynecological cancer survivors in Hong Kong. Psychooncology. 2002; 11(2):114-123.

21. Brotto LA, Yule M, Brecon E. Psychological interventions for the sexual sequelae of cancer: a review of the literature. J Cancer Surviv. 2010; 4:346-360.

22. Agarwal SS, Sehgal A, Sardana S, Kumar A, Luthra UK. Role of male behavior in cervical carcinogenesis among women with one-lifetime sexual partner. Health Educ Behav. Cancer. 1993; 72(5):1666-1669.

23. Wingood GM, Scd, DiClemente RJ. Application of the theory of gender and power to examine HIV-related exposures, risk factors, and effective interventions for women. Health Educ Behav. 2000; 27(5):539-565.

How to cite this article:

Ye S, Yang X, He G, Maciek PA, Zhou W. Consequences of cervical cancer treatment on sexual health in Chinese cancer survivors: a qualitative study. Glob Clin Transl Res. 2019; 1(1):31-36.

Copyright (c) 2019 by the Global Clinical and Translational Research 